\title{
CALIDAD DE ATENCIÓN EN SALUD REPRODUCTIVA Y PLANIFICACIÓN FAMILIAR CON ENFOQUE DE GÉNERO E INTERCULTURALIDAD EN LOS ESTABLECIMIENTOS DE SALUD DE CHUPACA Y JUSTICIA, PAZ Y VIDA - 2008*
}

\author{
Casallo Véliz, Soledad¹ , Pérez Gutarra, Guillermina² y Scarsi Maratuech, Rossana ${ }^{3}$ \\ Facultad de Enfermería de la Universidad Nacional del Centro del Perú
}

\begin{abstract}
RESUMEN
La investigación titulada "Calidad de atención en salud reproductiva y familiar planificación con enfoque de género e interculturalidad en los establecimientos de salud de Chupaca y Justicia, Paz y Vida" tuvo como objetivos principales determinar la calidad de atención de los servicios de salud reproductiva y planificación familiar; conocer la percepción de las usuarias sobre la calidad de atención recibida; determinar las barreras y oportunidades basadas en género e interculturalidad. El estudio es de tipo descriptivo analítico de corte transversal, tuvo como población los establecimientos de salud de Justicia Paz y Vida y Chupaca de la provincia de Huancayo. Los datos fueron obtenidos de la encuesta, entrevista semi estructurada y la observación. Los principales resultados fueron que la calidad de atención brindada en la estrategia de Salud Sexual y Reproductiva y Planificación Familiar no satisface plenamente las expectativas de las usuarias, no respondiendo a las necesidades de salud integral, con alternativas técnicas de alto nivel y respetuosas de sus patrones culturales; que garanticen la resolución del motivo de consulta en el momento oportuno y a un costo accesible; que tomen en cuenta los enfoques de interculturalidad y género; que propician su autoestima, autonomía, percepción y ejercicio del derecho a la salud y a decidir; y que promuevan la participación consciente y equitativa de los hombres en los procesos de salud sexual y reproductiva. Se evidencia la aplicación de normas, valores y políticas institucionales, pero requieren el involucramiento de todos los actores sociales para la construcción de una adecuada cultura organizacional que redunde en la buena calidad de atención en las dimensiones humanas, técnicas y del entorno.
\end{abstract}

Palabras clave: salud sexual y reproductiva, planificación familiar, calidad de atención, interculturalidad y género.

\section{"ATENCION QUALITY IN REPRODUCTIVE HEALTH AND FAMILIAR PLANNING WITH GENDER APPROACH AND INTERCULTURALIDAD IN THE ESTABLISHMENTS OF HEALTH OF CHUPACA AND JUSTICIA PAZ Y VIDA - 2008”}

\begin{abstract}
The research titled "Quality of attention in health reproductive and planning with approach of gender and interculturalidad in the health establishments of Chupaca and Justicia Paz y Vida" had as main targets to determine the health quality attention services of reproductive and familiar planning, to Know the perception of the users on the quality attention received; to determine the barriers and opportunities based in gender and interculturalism. The study is of a analytic descriptive type of transversal cut, had as population the health establishments of Justicia Paz y Vida - Huancayo, and Chupaca.The data were obtained of the survey, semi structured interview and the observation. The main results were that the attention quality offered in the strategy of Sexual Health and Reproductive and Familiar Planning does not satisfy fully the expectations of the users , not answering to the needs of integral health, with high level technical alternatives and respectful of their cultural patterns; that guarantee the resolution of the reason of query in the timely moment and to an accessible cost; that take in account the approaches of interculturalism and gender; To propiciate their self esteem, autonomy, perception and right to health and to decide; and that promote the conscious equitative participation of men in the processes of sexual and reproductive health.It was evidenced the application of norms, values and institutional politics, but they require the involvement of all the social actors to build an adecuated organizational culture which redound in a good quality attention in the human, technical and surroundings dimensions.
\end{abstract}

Key words: sexual health and reproductive, familiar planning, quality of attention, interculturalidad and generate.

\footnotetext{
* Este trabajo de investigación fue recibido el 20/11/2008 retornado para su revisión 26/10/2009 y aprobado para su publicación 16/11/2009

1Email:scasallov@yahoo.es

2Email:delia1999@hotmail.com

32Email:roscarsi76@yahoo.es
} 


\section{INTRODUCCIÓN}

En la presente investigación se determino la calidad de atención brindada en los servicios de salud reproductiva y planificación familiar, tomando como eje la estrategia nacional de salud sexual y reproductiva.

Desde hace muchos años ya, existe una creciente preocupación en todas las empresas de los países más avanzados por la búsqueda incesante de niveles más altos de calidad en sus productos y servicios. El origen de esta preocupación está claramente fundamentado en la evidencia de que la calidad ha cobrado un papel protagonista y constituye el factor fundamental de la competitividad de las empresas.

Considerando a los establecimientos de salud como una empresa productora de servicios sanitarios parece necesario también, desarrollar controles de calidad; si no por competitividad, sí por exigencias sociales o por imperativos profesionales. Conseguir un nivel de calidad óptimo equivale, por tanto, a desarrollar grados de efectividad, de eficiencia, de adecuación y de calidad científico-técnica en la práctica asistencial que satisfagan por igual a administradores, profesionales y usuarios.

Se caracterizara los factores que intervienen en la calidad del cuidado que brindan los profesionales considerando las dimensiones humanas, técnicas y del entrono como esenciales para evaluar la calidad de atención.

Los datos fueron obtenidos a través de la encuesta, la entrevista semiestructurada y la observación a través de la lista de cotejo. Teniendo como objetivos: Determinar la calidad de atención de los servicios de salud reproductiva y planificación familiar; Conocer la percepción de las usuarias sobre la calidad de atención recibida en los servicios ;identificar las barreras y oportunidades basadas en género e interculturalidad en los servicios de salud reproductiva y planificación familiar de los establecimientos de estudio; valorar el nivel de cumplimiento en la protección y promoción de los derechos de la usuaria por parte de prestadores.

\section{MATERIALES Y MÉTODOS}

\section{Método de Investigación}

Estudio analítico de corte transversal

\section{Diseño metodológico}

\section{- Población y muestra}

El estudio ha sido aplicado en dos establecimientos de salud de la Región Junín: Centro de Salud de Chupaca y Centro de Salud (ACLAS) de Justicia, Paz y Vida. Como población se consideró a 3 obstétricas de cada establecimiento. Las usuarias encuestadas/ entrevistadás fueron las que asistieron a su atención en la estrategia de salud sexual y reproductiva y planificación familiar, hasta llegar a un número de 30 en cada establecimiento.

\section{Procesamiento y análisis de datos}

Se aplicó la encuesta, cuestionario semiestructurado y lista de cotejo, qué cubren las áreas a investigar, los datos han sido vaciados al programa excel y se procedió a hacer, los cuadros estadísticos para la interpretación y análisis respectivo.

\section{RESULTADOS Y DISCUSIÓN}

Tabla 1. El personal de salud brinda cuidados al cliente con competencia técnica

\begin{tabular}{ccc}
\hline CUIDADOS & № & $\%$ \\
\hline Frecuentemente & 3 & 50 \\
De vez en cuando & 3 & 50 \\
Muy rara vez & 0 & 0 \\
Total & 6 & 100 \\
\hline
\end{tabular}

Fuente: Encuesta aplicada por los investigadores 2008

\section{BRINDA CUIDADO INTEGRAL CON CALIDAD Y CALIDEZ}

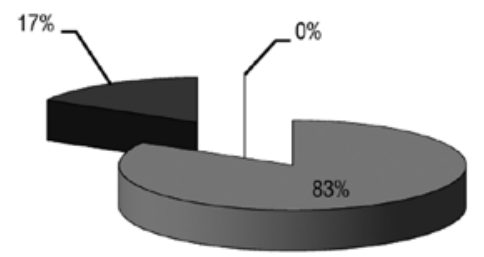

Gráfico 1. Brinda cuidado integral con calidad y calidez 
Las organizaciones en general deben dar las bases para que sus colaboradores tengan la preparación necesaria y especializada que les permitan enfrentarse en las mejores condiciones a sus tareas diarias. Sin embargo se puede evidenciar que la capacitación en el ACLAS Justicia, Paz y Vida y Centro de Salud de Chupaca es mínima, no asegurando el adecuado desarrollo de competencias del personal, lo cual tendrá impacto en la capacidad técnica.

En relación a la calidad y calidez, el $83 \%$ de los encuestados refieren que es brindada frecuentemente en las diferentes actividades que realizan, hecho que no se corrobora en su totalidad al confrontar las respuestas de los usuarios sobre el tema.

EL PERSONAL DE SALUD CONSIDERA QUE EN SU ESTABLECIMIENTOSE PRACTICA VALORES INSTITUCIONALES

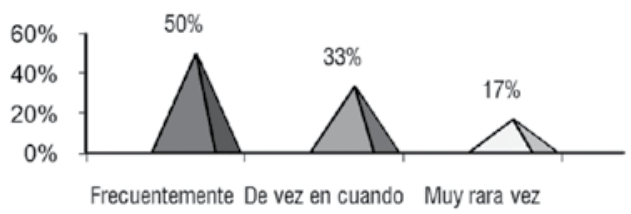

Gráfico 2. El personal de salud considera que en su establecimiento se practican valores institucionales.

OPINIÓN DEL PERSONAL SOBRE EVIDENCIAS DE TRABAJO EN EQUIPO

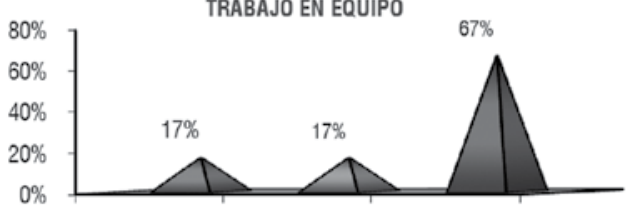

FrecuentementeDe vez en cuando Muy rara vez

Gráfico 3. Opinión del personal sobre evidencias de trabajo en equipo
OPINIÓN DEL PERSONAL SOBRE LAS RELACIONES INTERPERSONALES EN LA INSTITUCIÓN

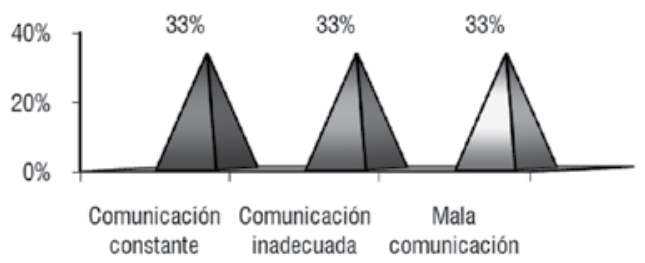

Grafico 4. Opinión del personal sobre las relaciones interpersonales en la institución

Los valores en una organización son considerados como la personalidad de la misma, la forma como se comportará y como vivirá; son en sí parte del espíritu de la organización, un fiel reflejo de su líder.

Conocer y atender la problemática de los valores permite detectar los conflictos y solucionarlos. En gran medida el funcionamiento de la organización está determinado por los valores y el trabajo equipo, los cuales deben funcionar como un sistema operativo y de orientación indicando de forma adecuada como solucionar las necesidades e incluso la prioridad que se debe dar a cada uno, todo ello conlleva a mejorar el clima organizacional y las relaciones interpersonales en el establecimiento.

En los establecimientos de estudio, se pudo evidenciar indicios de trabajo en equipo y aplicación de valores siendo necesario de parte de los actores sociales la identificación de nuevos valores y actitudes que favorezcan su productividad y fortalezcan las relaciones interpersonales para lograr una cultura organizacional fuerte orientada a la persona que redunde en una adecuada calidad de atención al usuario y permita que los trabajadores se encuentren motivados.

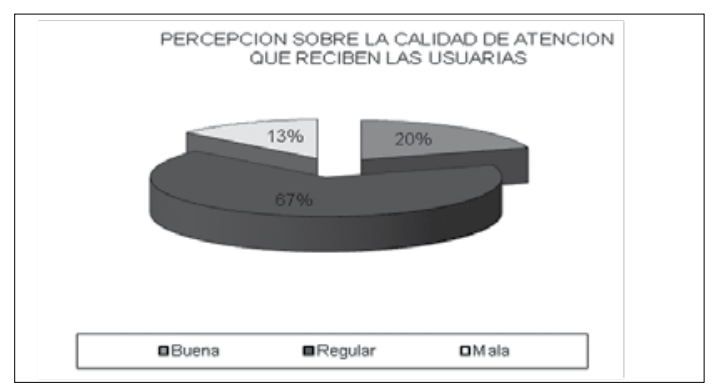

Gráfico 5. Percepción sobre la calidad de atención que reciben las usuarias 
PERCEPCION DE LA USUARIA SOBRE LAS CARACTERISTICASDEL PERSONAL DE SALUD

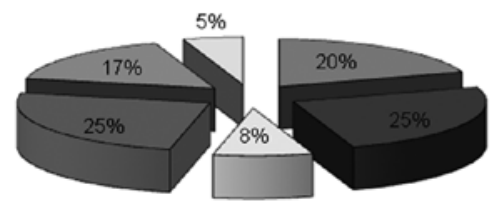

$\begin{array}{lll}\text { QAtento } & \text { QAmable } & \text { QCarińoso } \\ \text { aMal humorado } & \text { aNo tratable } & \text { QApurado }\end{array}$

Gráfico 6. Percepción de la usuaria sobre las características del personal de salud.

\section{PERCEPCION DE LA USUARIA EN RELACION A LA ACLARACION DE DUDAS}

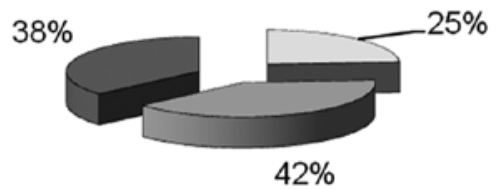

Gráfico 7. Percepción de la usuaria en relación a la aclaración de dudas.

Tabla 2. Percepción de la usuaria en relación a la consejería brindada por el personal

\begin{tabular}{ccc}
\hline PERCEPCIÓN & № & $\%$ \\
\hline Siempre & 14 & 23 \\
Casi siempre & 16 & 27 \\
Nunca & 30 & 50 \\
TOTAL & 60 & 100
\end{tabular}

Fuente: Encuesta aplicada por los investigadores 2008

El $67 \%$ opina que la atención recibida es de regular calidad, teniendo un $8 \%$ que opina que es de mala calidad. En relación a la percepción de las características del personal de salud, el 25\% opina que el personal que la atiende es mal humorado, el $17 \%$ no tratable y el $5 \%$ apurado, así mismo el $38 \%$ refiere que el personal no aclara sus dudas generando barreras de accesibilidad. En este con- texto es necesario mencionar que, para acercar a la población a los establecimientos de salud y eliminar las barreras de acceso, el Ministerio de Salud (MINSA) estableció nuevas medidas para mejorar la orientación y consejería en materia de salud sexual y reproductiva, específicamente en planificación familiar.

En este contexto se considera que la mayoría de usuarios podrá acceder a la información necesaria sobre salud sexual y reproductiva y planificación familiar para tomar decisiones libres e informadas. Así mismo en los establecimientos de estudio se puede evidenciar la falta de mecanismos de motivación y de condiciones favorables para una buena calidad de atención, sin embargo hay un buen porcentaje de respuestas que evidencian el esfuerzo por cumplir con estándares adecuados de atención y consejería, siendo necesario fortalecer mecanismos que permitan implementar un sistema de motivación y desarrollo del capital humano para que puedan lograr competencias para una adecuada consejería/ información en salud sexual y reproductiva y planificación familiar.

También se requiere de mecanismos que permitan mejorar el trato a las usuarias, ya que se evidencia un alto porcentaje de respuestas en el que las usuarias refieren mal trato, falta de confianza e inadecuada calidad de atención.

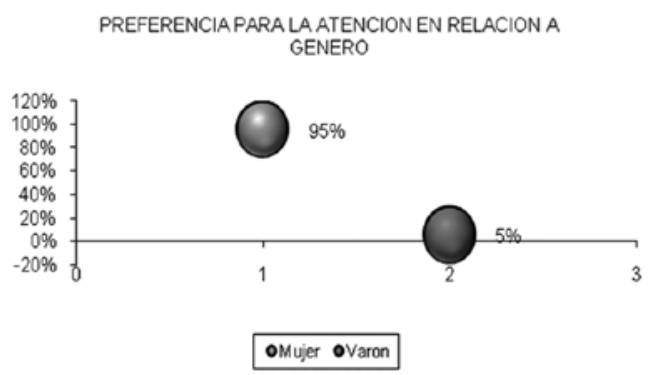

Grafico 8. Preferencia de la usuaria para su atención en relación a género 
INFORMACION SOBRE SALUD SEXUAL Y REPRODUCTIVA

PARA VARONES

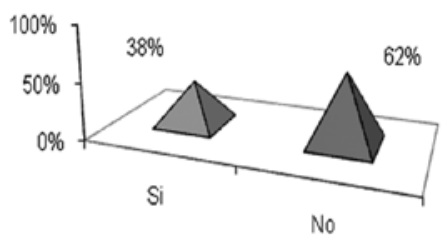

aSi aNo

Gráfico 9. Existen actividades de información sobre salud sexual y reproductiva para los varones.

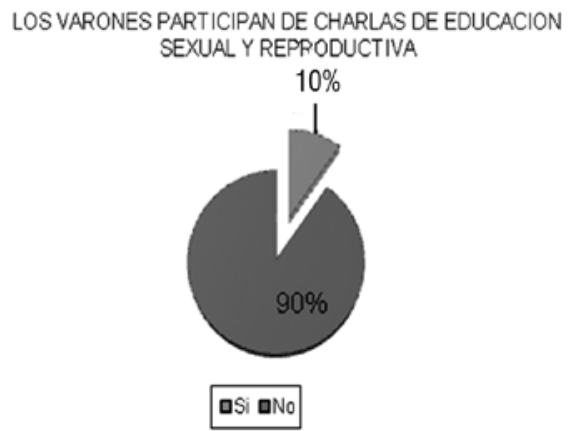

Gráfico 10. Los varones participan activamente de charlas de salud sexual y reproductiva

Aplicar el enfoque de género permite reconocer la identidad que asumen hombres y mujeres derivada de la asignación de los roles impuestos a partir de su sexo y acordes a su contexto sociocultural, que al ser introducidos determinan muchos aspectos de su conducta y en el ejercicio del poder a todos niveles, generando desigualdad e inequidad en las condiciones de vida, donde con mayor frecuencia son las mujeres las menos favorecidas.

En los cuadros se puede evidenciar la participación mínima de varones en salud sexual y reproductiva y planificación familiar siendo necesario planear acciones para que los procesos de atención propicien la participación tanto de la mujer como del varón y sean más efectivos promoviendo desde los diferentes ámbitos la igualdad y la equidad entre hombres y mujeres.

\section{Derechos en Salud:}

LOSUSUARIOS CONOCEN SUS DERECHOS EN SALUD

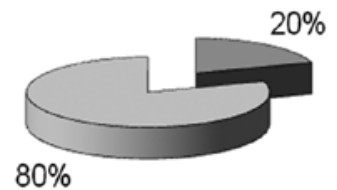

Gráfico 11. Los usuarios conocen sus derechos en salud.

Tabla 3. Opinión de las usuarias en relación a si el personal respeta sus creencias

\begin{tabular}{lcc}
\hline RESPETA LAS CREENCIAS & № & $\%$ \\
\hline Si & 14 & 23 \\
No & 46 & 77 \\
TOTAL & 60 & 100 \\
\hline
\end{tabular}

Fuente: Encuesta aplicada por los investigadores 2008

Podemos observar que el $77 \%$ de usuarias refieren que en los establecimien tos de estudio no le toman interés a sus creencias a pesar que la interculturalidad es uno de los principales enfoques del sector salud, orientado a lograr una interacción entre culturas, de una forma respetuosa, horizontal y sinérgica, donde se concibe que ningún grupo cultural está por encima del otro, favoreciendo en todo momento la integración y convivencia de ambas partes logrando las relaciones interculturales que permitan mejorar la accesibilidad a los servicios de salud a través del dialogo, la escucha mutua, la concertación ,la sinergia y respeto de las formas de pensar.

En las relaciones interculturales se establece una relación basada en el respeto a la diversidad y el enriquecimiento mutuo; sin embargo no es un proceso exento de conflictos, estos se resuelven mediante el respeto, el diálogo, la escucha mutua, la concertación y la sinergia.

En este contexto los establecimientos de salud deben de brindar una atención basada en enfoques de género, interculturalidad y derechos en salud. 


\section{CONCLUSIONES}

- En los Servicios de Salud de Justicia, Paz y Vida y Chupaca se pudo evidenciar que la calidad de atención brindada en la estrategia de salud sexual y reproductiva y planificación familiar no satisface plenamente las expectativas de las usuarias no respondiendo a las necesidades de salud integral, con alternativas técnicas de alto nivel y respetuosas de sus patrones culturales; que garanticen la resolución del motivo de consulta en el momento oportuno y a un costo accesible; que tomen en cuenta el conocimiento que tienen las mujeres sobre sus procesos de salud; que propician su autoestima, autonomía, percepción y ejercicio del derecho a la salud y a decidir; y que promueven la participación consciente y equitativa de los hombres en los procesos de salud sexual y reproductiva.

- Considerando que el capital humano (prestador) cumple un rol estratégico en todo el proceso de cambio ya que es responsable de implementar las normas, políticas y propuestas diseñadas para un determinado objetivo y por sobre todo brindar una atención de calidad y calidez; requiriendo de una preparación adecuada, se puede evidenciar que la mayoría de ellos no han recibido capacitación en temas relacionados a salud sexual y reproductiva. En el estudio se pudo evidenciar que el $50 \%$ del personal entrevistado refiere que muy rara vez participa en cursos de capacitación.

- En relación a la disponibilidad de equipamiento y abastecimiento de insumos existen limitaciones que impiden una atención sin riesgos para la salud. Al respecto el $67 \%$ del personal considera que los equipos se encuentran en regular estado de conservación, hecho que se corroboró con la observación.

- Se evidencia que ambos establecimientos cuentan con normas, políticas y valores institucionales, los cuáles permiten afianzar proceso de cambio en mejora de la calidad de atención. Así mismo se evidencia indicios de trabajo en equipo y adecuadas relaciones interpersonales.

- La calidad humana es una de las dimensiones de la calidad de atención, sin embargo se pudo evidenciar que el $25 \%$ de las usuarias refieren que el personal normalmente está mal humorado, el 17\% no tratable, sin embargo en cifras similares las usuarias refieren que el trato es amable.

- No existe seguimiento ni ruta establecida para brindad servicios extramurales.

- No se brinda información sobre derechos sexuales y reproductivos, evidenciándose que las usuarias tienen escaso conocimientos sobre el tema. El $80 \%$ de ellas refieren que no conocen sus derechos como usuarias, lo que de alguna manera impide el ejercicio de los mismos.

- El enfoque de género e interculturalidad es aplicado en forma insipiente en ambos establecimientos, evidenciándose la mínima participación del varón y la falta de aceptación de las creencias y costumbres de las usuarias, impidiendo lograr una adecuada interacción en forma respetuosa, horizontal y simétrica, impidiendo una adecuada calidad de atención.

\section{LITERATURA CITADA}

CEDEMU. 2002. Sentando bases para el desarrollo de un modelo intercultural en salud sexual y reproductiva un estudio de epidemiología intercultural, balance del proceso. Arica: CEDEMU.

Citarella L. 2000 . (comp.) Medicinas y culturas en la Araucanía. Santiago de Chile: Editorial Sudamericana.

Ibacache J, Oyarce AM. 1996. Reflexiones para una política intercultural en salud. Puerto Saavedra, Chile: Primer encuentro Nacional de salud y Pueblos Indígenas.

Informe de la Conferencia Internacional sobre la Población y el Desarrollo. 1994. El Cairo, 5 a 13 de septiembre. (Sitio en Internet) Disponible en: www.cinu.otg.mx/temas/desarrollo/dessocial/ población/icpdf1994.htm

MINSA. 2005. Manual de normas de atención en salud sexual y reproductiva. 
Varo J. 1994.Gestión estratégica de la calidad en los servicios sanitarios. Un modelo de gestión hospitalaria. Ediciones Díaz de Santos. S.A. Madrid.

Velloso A. y Trevisan M. 1998. Gerenciamiento de la calidad en enfermería Rev. Informativo Latinoamericano de Enfermería. 25/26: 13.

Leddy S. y Pepper J. 1989 Bases conceptuales de la enfermería. Organización Panamericana de la Salud. New York. U.S.A.
Pabón, H. 1987.Evaluación de los servicios de salud. Universidad del Valle. Facultad de Salud. PRIDE. Colombia.

Salinas C, Laguna J, Mendoza M. 1996. La satisfacción laboral y su papel en la evaluación de la calidad de la atención médica. Salud Pública de México. Volumen 36, № 1 